\title{
EFECTO HIPOTENSOR DEL EXTRACTO DE AJO (Allium sativum) MACERADO POR 18 SEMANAS EN UN MODELO EXPERIMENTAL IN VIVO
}

\author{
David Chaupis-Meza1,2,a, Juan Rojas ${ }^{3, b}$, Manuel Gasco ${ }^{4, c}$, Gustavo F. Gonzales
}

\begin{abstract}
RESUMEN
Objetivos. Determinar si el extracto de ajo (Allium sativum) macerado por 18 semanas tiene igual o mejor efecto hipotensor que el captopril en ratas. Materiales y métodos. Se realizó un estudio experimental in vivo con ratas machos Holtzman, clasificados en cinco grupos: 100,500 y $1000 \mathrm{mg} / \mathrm{kg}$ de extracto de ajo, Captopril de $100 \mathrm{mg} / \mathrm{kg}$ y un grupo vehículo. El L-NAME ( $\mathrm{N}_{\mathrm{G}^{-}}$-nitro L-arginina-metil-éster) administrado vía intraperitoneal $50 \mathrm{mg} / \mathrm{kg}$ desde el inicio del experimento, elevó la presión arterial desde el tercer día. El análisis estadístico consistió en las pruebas T de Student para medias pareadas, ANOVA y comparación múltiple de Scheffe. Resultados. El ajo macerado extraído por un proceso hidroalcohólico durante 18 semanas provocó una disminución de la presión arterial en animales de experimentación. El análisis de los tratamientos sobre la presión arterial media (PAM), obtuvieron diferencias significativas desde el tercer día. La comparación sobre la PAM final versus PAM basal (medias no diferentes) y el efecto hipotensor (\%) fueron: ajo-100 ( $p=0,008), 59,8 \%$; ajo-500 ( $p=0,021), 80,6 \%$; ajo-1000 ( $p=0,034), 88,5 \%$, Captopril $(p=0,437), 99,9 \%$ y vehículo $(p=0,001), 0 \%$. Conclusiones. El ajo macerado a un periodo de 18 semanas resultó eficaz para producir un efecto hipotensor en ratas, inducidas a hipertensión arterial por L-NAME.
\end{abstract}

Palabras clave: Ajo; Compuestos fenólicos; Enfermedad crónica; Hipertensión; Ratas; L-NAME (fuente: DeCS/BIREME).

\section{HYPOTENSIVE EFFECT OF EXTRACT OF MACERATED GARLIC (Allium sativum) FOR 18 WEEKS IN AN IN VIVO EXPERIMENTAL MODEL}

\begin{abstract}
Objectives. Determine whether macerated extract of garlic (Allium sativum) for 18 weeks is equal to or better than Captopril in its hypotensive effect in rats. Materials and methods. We performed an experimental in vivo study with Holtzman male rats divided into five groups using 100, 500 and 1,000 mg kg of garlic extract, Captopril $100 \mathrm{mg} / \mathrm{kg}$ and a vehicle group. $50 \mathrm{mg} / \mathrm{kg} \mathrm{L-NAME} \mathrm{(NG-nitro-L-arginine} \mathrm{methyl} \mathrm{ester)} \mathrm{was} \mathrm{administered} \mathrm{intraperitoneally} \mathrm{which} \mathrm{elevated}$ blood pressure after the third day. Statistical analysis consisted of Student's t-test for paired means, ANOVA and Scheffe multiple comparison. Results. The macerated garlic extracted by a hydroalcoholic process administered for 18 weeks resulted in a decrease in blood pressure in experimental animals. In the analysis of treatments on mean arterial pressure (MAP), significant differences were obtained after the third day. The comparison of the MAP final versus MAP basal (no difference in averages) and the hypotensive effect (\%) were: garlic-100 ( $p=0.008), 59.8 \%$; ajo-500 ( $p=0.021), 80.6 \%$; garlic-1000 ( $p=0.034), 88.5 \%$; captopril $(p=0.437), 99.9 \%$; and vehicle-only $(p=0.001), 0 \%$. Conclusions. The macerated garlic given for an 18-week period effectively produced a hypotensive effect in rats with hypertension induced by L-NAME.
\end{abstract}

Key words: Garlic; Phenolic compounds; Chronic disease; Hypertension; Rats; L-NAME (source: MeSH/NLM).

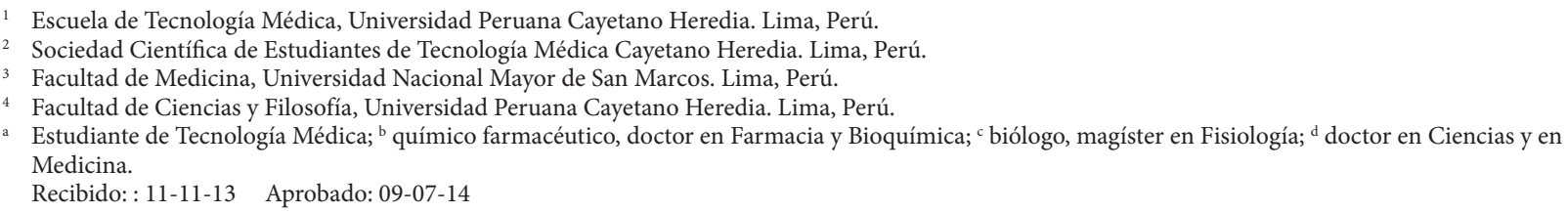

Citar como: Chaupis-Meza D, Rojas J, Gasco M, Gonzales GF. Efecto hipotensor del extracto de ajo (Allium sativum) macerado por 18 semanas en un modelo experimental in vivo. Rev Peru Med Exp Salud Publica. 2014;31(3):461-66. 


\section{INTRODUCCIÓN}

Las enfermedades crónicas no transmisibles (ECNT) se han constituido en el principal problema de salud pública a nivel mundial (1). Se estima que para el 2020 las ECNT causarán el $80 \%$ de cargas globales sanitarias, provocando la muerte de siete de cada diez personas en países en vías de desarrollo ${ }^{(2)}$. Asimismo, las enfermedades cardiovasculares, entre ellas la hipertensión arterial (HTA), presenta aproximadamente 9,4 millones de muertes al año, mientras que en las Américas la prevalencia es del $35 \%{ }^{(3)}$. En el Perú, la prevalencia de HTA ascendió de 23,7 a $27,3 \%{ }^{(4,5)}$, en solo cinco años.

El impacto que la HTA genera en la salud de muchas personas se ve complicado por la falta de conocimiento que tienen sobre su propia condición. Actualmente, solo el $48,2 \%$ de personas hipertensas en el Perú conocen su enfermedad, de estas, el $81,5 \%$ recibe algún tratamiento ${ }^{(4)}$. Pese a existir un elevado control de la HTA a nivel nacional, la automedicación sigue siendo un problema en nuestra población ${ }^{(6)}$. Entre tanto, existe la demanda por incurrir al diseño de alternativas terapéuticas mejoradas de bajo costo. En tal sentido, los productos naturales son comúnmente utilizados como medicina tradicional en diversos lugares del mundo. Especies como el ajo viene usándose desde tiempos remotos para reducir la presión arterial (7), distintos estudios aseveran su efecto hipotensor ${ }^{(8-12)}$.

Se ha explorado la posibilidad de potenciar el efecto hipotensor del ajo, mediante técnicas de maceración. Hoy en día existen dos técnicas basadas en el tiempo de maceración, la primera, denominada extracción añeja de ajo (EAA) en un tiempo aproximado de 20 meses a temperatura ambiente, ha sido utilizada en diversos estudios (13-16); y la segunda técnica, denominada fermentación espontanea a corto plazo (FECP) en un tiempo de 40 días bajo $60-70{ }^{\circ} \mathrm{C}{ }^{(17-18)}$. Ambas técnicas se basan en la descomposición que sufren los órganos sulfurados del ajo, como la aliina (compuesto de aminoácidos azufrados) que al ser triturado se transforma por la alicinasa en alicina, este inestable componente se descompone en s-alil-cisteína (SAC) al ser ingerido, además la reacción tiene como subproducto el ácido pirúvico. Este último en adición con el triptófano, se obtiene la 1,2,3,4-tetrahidro- $\beta$-carbolina $(\mathrm{TH} \beta \mathrm{C}){ }^{(19)}$, potente antioxidante sobre la síntesis de óxido nítrico (ON) molécula encargada de conseguir la vasodilatación arterial y con ello la disminución de la presión sanguínea ${ }^{(20)}$. El presente estudio combinó ambas técnicas de maceración de ajo, con el objetivo de evaluar si en un tiempo de 18 semanas tiene igual o mejor efecto hipotensor que el captopril. El estudio fue llevado a cabo en un modelo experimental en animales, inducidos a hipertensión por L-NAME $\left(\mathrm{N}_{-}{ }_{-}^{-}\right.$ nitro L-arginina metil éster). Este estudio constituye una inicial exploración in vivo.

\section{MATERIALES Y MÉTODOS}

\section{DISEÑO DEL ESTUDIO}

Estudio realizado de abril de 2012 a agosto de 2013 en las instalaciones de la Universidad Peruana Cayetano Heredia en colaboración técnica con la Facultad de Medicina de la Universidad Nacional Mayor de San Marcos. El diseño fue de tipo experimental in vivo.

\section{ANIMALES}

Se utilizaron ratas adultas machos de la cepa Holtzman con un peso de $200 \pm 10 \mathrm{~g}$, obtenidos del bioterio de la Universidad Peruana Cayetano Heredia. Los animales de experimentación fueron agrupadas en cinco jaulas de siete animales por jaula, a temperatura de $23{ }^{\circ} \mathrm{C}$ con un ciclo de luz/oscuridad de 12:12 horas. Fueron alimentadas con Purina ${ }^{\circledR}$ y agua de filtro ad libitum.

\section{PREPARACIÓN DE LA EXTRACCIÓN DE AJO MACERADO A MEDIANO TIEMPO}

Los ajos blancos utilizados en la preparación del extracto fueron obtenidos de un proveedor local, procurando que estén en buenas condiciones. Asimismo, el Laboratorio de Botánica Aplicada del Departamento de Ciencias Fisiológicas y Farmacéuticas de la Universidad Peruana Cayetano Heredia identificó la muestra de ajo como: bulbos de ajos blancos (Allium sativum, familia: Liliaceae), sin voucher de registro. Para efecto del estudio solo se utilizaron los bulbos de ajo, siendo estas las principales partes en donde se obtendría la actividad biológica de este producto.

La preparación del extracto se hizo sobre la base de la las técnicas de EAA y FECP. Los ajos $(500 \mathrm{~g})$ fueron suspendidos en una solución hidroalcohólica $(1500 \mathrm{~mL}$, al $75 \%$ ), previamente, fueron pelados y cortados en rodajas y almacenados por 40 días a una temperatura entre $60-70{ }^{\circ} \mathrm{C}$, posterior a ello, se mantuvieron suspendidos en una maceración anaerobia por 18 semanas. Al término de la maceración se procedió a filtrar la solución y llevarla a un rotavapor R-114 $\left(B \cup C H I^{\circledR}\right.$, Switzerland) para su extracción al vacío y, finalmente, se logró extraer $855 \mathrm{mg}$ que se mantuvo en refrigeración a $4{ }^{\circ} \mathrm{C}$. 


\section{INDUCCIÓN Y TRATAMIENTO DE LA HIPERTENSIÓN ARTERIAL}

Los cinco grupos de ratas tratadas tuvieron el siguiente orden: Grupo AJO1: extracción de ajo de $100 \mathrm{mg} / \mathrm{kg}$; Grupo AJO2: extracción de ajo de $500 \mathrm{mg} / \mathrm{kg}$; Grupo AJO3: extracción de ajo de $1000 \mathrm{mg} / \mathrm{kg}$; Grupo control positivo: captopril de $100 \mathrm{mg} / \mathrm{kg}$; Grupo control negativo: vehículo.

Todos los tratamientos fueron diluidos con $0,2 \mathrm{~mL}$ de dimetilSulfoxido (DMSO), al 50\% (vehículo). El experimento duró 10 días, fueron 35 ratas a las que se les indujo HTA con L-NAME (Sigma ${ }^{\circledR}$ ) inyectando $50 \mathrm{mg} / \mathrm{kg}$ vía intraperitoneal, durante todo el periodo de experimentación, siguiendo el método utilizado por Pereira et al. (21) y Rojas et al. ${ }^{(22)}$. Los tratamientos con ajo, captopril y el vehículo, comenzaron a partir del tercer día, siendo administrados por vía oral (1 $\mathrm{mL}$ ) empleando una sonda orogástrica $\mathrm{N} .^{\circ} 18$ para la inoculación.

\section{MEDICIÓN DE LA PRESIÓN ARTERIAL}

La presión arterial fue evaluada mediante la técnica de tail-cuff (presión arterial indirecta), con el aparato LE 5002 (Letica ${ }^{\circledR}$ ) equipado con un microprocesador, envuelto en un pequeño brazalete, el cual detecta la presión de la arteria caudal ubicada en la cola de la rata. Los animales fueron ubicados dentro de un cepo acrílico, el cual solo exponía la cola, la cual por convección mediante una cámara térmica se pudo vasodilatar por un promedio de 15 minutos por rata, ello permitió una adecuada valoración de las presiones arteriales.

Las evaluaciones se dieron por triplicado por cada rata, luego se promediaron los resultados obtenidos, dichas evaluaciones se realizaron durante todo el experimento, se registraron cinco evaluaciones de la siguiente manera: DO (basal): primer día de evaluación; D2: tercer día de evaluación; D5: sexto día de evaluación; D7: octavo día de evaluación; D9 (final): décimo día de evaluación.

Las evaluaciones se llevaron siempre a la misma hora (09.00 h); antes de cualquier inducción y/o inoculación se procuraba que los animales estén lo menos estresados.

\section{ANÁLISIS ESTADÍSTICO}

Los datos son presentados según su escala de medición: como media y desviación estándar (DE), para valores en la escala de razón. Luego de determinar los supuestos estadísticos (prueba de Shapiro Wilk para comprobar la distribución normal, prueba de Bartlett para la homogeneidad y una prueba de aleatoriedad), se procedió a utilizar el análisis de varianzas de una vía (ANOVA) y una prueba de comparación múltiple como Scheffe. Se decidió utilizar la prueba T pareada para determinar los resultados de la presión arterial media (final versus basal); asimismo, se utilizaron promedios porcentuales para una mejor descripción de los resultados. La fórmula que se utilizó para determinar el efecto hipotensor, fue la siguiente: efecto hipotensor (\%) $=(\mathrm{Cp}-\mathrm{Tp}) / \mathrm{Cp} \times 100$. Donde $\mathrm{Cp}$ determina el promedio de las diferencias del grupo vehículo, es decir PAM final - PAM basal, asimismo, Tp se trata del promedio de las diferencias de cada grupo de ajo añejado y captopril. Los resultados fueron analizados utilizando el paquete estadístico Stata versión 12.

\section{ASPECTOS ÉTICOS}

Al final del estudio los animales, fueron sacrificados con una sobredosis de pentobarbital sódico. Los experimentos llevados a cabo en los animales estuvieron sujetos a la "Guide for the care and use of laboratory animals» de la National Institutes of Health de los Estados Unidos ${ }^{(23)}$. El Comité Institucional de Ética en Animales, de la Universidad Peruana Cayetano Heredia aprobó el presente estudio.

\section{RESULTADOS}

El tratamiento con L-NAME generó un incremento sustancial sobre la presión arterial, lo cual se logró evidenciar al tercer día de evaluación. Durante el tiempo del tratamiento no presentaron ningún tipo de reacciones adversas, ni se produjeron decesos en los animales.

Los hallazgos mostraron que los tratamientos en base a ajo lograron reducir la presión arterial, en comparación con el fármaco estándar captopril y el vehículo. Esto se presenció en los distintos tipos de presiones registradas como la presión arterial sistólica (PS), presión arterial diastólica (PD) y la presión arterial media (PAM), para cada grupo de tratamiento, evidenciado a partir del tercer día, asimismo, se encontró relación con el día en que se empezaron los tratamientos. Sin embargo, dichas diferencias parecen no presentarse en todos los grupos con respecto al vehículo, ya que al realizar una prueba de comparación múltiple de Scheffe se pudo demostrar que las diferencias entre los grupos de interés contra el grupo vehículo resultaron significativos a partir del día $7(p>0,05)$, el grupo captopril adquirió diferencia significativa versus el grupo vehículo desde el día $5(p<0,05)$. Finalmente, el grupo de ajo $1000 \mathrm{mg} / \mathrm{kg}$ frente al grupo captopril no obtuvieron diferencias significativas (Tabla 1). 
Tabla 1. Variación de las presiones arteriales, según día de tratamiento

\begin{tabular}{|c|c|c|c|}
\hline $\begin{array}{l}\text { Tratamiento } \\
\text { según día }\end{array}$ & 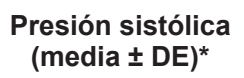 & $\begin{array}{l}\text { Presión diastólica } \\
\quad(\text { media } \pm D E)^{*}\end{array}$ & $\begin{array}{l}\text { Presión arterial media } \\
\quad(\text { mediana } \pm \mathrm{DI})^{\star *}\end{array}$ \\
\hline \multicolumn{4}{|l|}{ Ajo 100} \\
\hline Día 0 & $125,37 \pm 5,29$ & $92,60 \pm 3,96$ & $104,27 \pm 1,87$ \\
\hline Día $2^{\dagger}$ & $170,66 \pm 2,89$ & $112,26 \pm 8,67$ & $129,40 \pm 2,15$ \\
\hline Día 5 & $171,94 \pm 3,29^{b}$ & $116,11 \pm 8,01^{b}$ & $135,07 \pm 1,72^{b}$ \\
\hline Día 7 & $154,97 \pm 3,99 \mathrm{a}, \mathrm{b}$ & $117,46 \pm 4,90^{a, b}$ & $129,33 \pm 1,48^{a, b}$ \\
\hline Día 9 & $145,03 \pm 5,76^{a, b}$ & $114,89 \pm 6,13^{a, b}$ & $123,33 \pm 3,90^{a, b}$ \\
\hline \multicolumn{4}{|l|}{ Ajo 500} \\
\hline Día 0 & $129,29 \pm 6,18$ & $96,57 \pm 9,07$ & $105,67 \pm 4,30$ \\
\hline Día $2^{\dagger}$ & $170,14 \pm 4,18$ & $107,97 \pm 4,24$ & $128,80 \pm 1,53$ \\
\hline Día 5 & $164,57 \pm 1,57$ & $114,17 \pm 3,98$ & $131,93 \pm 1,07$ \\
\hline Día 7 & $140,80 \pm 3,75^{a}$ & $116,17 \pm 3,82^{a}$ & $123,40 \pm 1,95^{a}$ \\
\hline Día 9 & $135,66 \pm 1,97$ a,b & $104,71 \pm 2,40^{a, b}$ & $114,87 \pm 0,80^{a, b}$ \\
\hline \multicolumn{4}{|l|}{ Ajo 1000} \\
\hline Día 0 & $124,26 \pm 5,22$ & $89,77 \pm 6,86$ & $101,53 \pm 1,32$ \\
\hline Día $2^{\dagger}$ & $162,63 \pm 2,78$ & $113,43 \pm 5,56$ & $128,73 \pm 1,65$ \\
\hline Día 5 & $158,43 \pm 7,71$ & $117,63 \pm 6,10$ & $131,27 \pm 4,10$ \\
\hline Día 7 & $137,80 \pm 6,87$ & $111,94 \pm 6,51$ & $119,40 \pm 1,17^{a}$ \\
\hline Día 9 & $132,69 \pm 1,62^{a}$ & $94,20 \pm 2,65^{a}$ & $107,00 \pm 0,97^{a}$ \\
\hline \multicolumn{4}{|l|}{ Captopril } \\
\hline Día 0 & $122,46 \pm 10,34$ & $90,66 \pm 8,61$ & $103,27 \pm 3,80$ \\
\hline Día $2^{\dagger}$ & $160,91 \pm 2,22$ & $112,00 \pm 4,89$ & $127,80 \pm 1,13$ \\
\hline Día 5 & $157,83 \pm 6,69^{a}$ & $112,09 \pm 1,11^{a}$ & $128,07 \pm 0,83^{a}$ \\
\hline Día 7 & $135,71 \pm 1,77^{a}$ & $108,89 \pm 4,22^{a}$ & $116,80 \pm 1,87^{a}$ \\
\hline Día 9 & $128,83 \pm 3,72^{a}$ & $90,57 \pm 2,07^{a}$ & $103,33 \pm 1,52^{\mathrm{a}}$ \\
\hline \multicolumn{4}{|l|}{ Vehículo } \\
\hline Día 0 & $121,91 \pm 8,75$ & $92,77 \pm 2,98$ & $101,13 \pm 3,08$ \\
\hline Día $2^{\dagger}$ & $170,00 \pm 1,53$ & $109,60 \pm 4,25$ & $129,87 \pm 1,62$ \\
\hline Día 5 & $179,69 \pm 4,40$ & $113,86 \pm 3,90$ & $136,27 \pm 1,43$ \\
\hline Día 7 & $186,89 \pm 2,59$ & $118,09 \pm 11,10$ & $141,53 \pm 6,02$ \\
\hline Día 9 & $186,14 \pm 4,02$ & $129,74 \pm 4,26$ & $148,53 \pm 1,27$ \\
\hline
\end{tabular}

*(media \pm Desviación Estándar), ${ }^{* *}($ mediana \pm Desviación Intercuartil $), \dagger$ Día de inicio de tratamiento

a $p<0,05$, con respecto al vehículo, ${ }^{\mathrm{b}} p<0,05$, con respecto al grupo Captopril.

Los promedios porcentuales hallados para el efecto hipotensor haciendo comparaciones de medias entre muestras pareadas (Día 0 y Día D9) de cada tratamiento, fueron: ajo de $100 \mathrm{mg} / \mathrm{kg}$ igual a $53,5 \%(p=0,01)$; ajo de $500 \mathrm{mg} / \mathrm{kg}$ igual a $83,6 \%(p=0,02)$; ajo de $1000 \mathrm{mg} / \mathrm{kg}$ igual a $87,5 \%(p=0,03)$; captopril de $100 \mathrm{mg} / \mathrm{kg}$ igual a $92,5 \%(p=0,43)$; vehículo igual a $0 \%(p=0,01)$.

\section{DISCUSIÓN}

Los resultados del estudio permiten evidenciar el efecto hipotensor del ajo macerado a mediano tiempo, en distintas dosis de evaluación. De tal manera, el incremento en la dosificación de los tratamientos basados en ajo produce una mayor respuesta hipotensora tan cercano al efecto alcanzado por el fármaco estándar captopril.
Según otros estudios, existiría la presencia de metabolitos implicados en el efectos hipotensor tal como la TH $\beta C$ s, hallada en un estudio realizado por Ichikawa et al. en el $2006{ }^{(19)}$, demostrando ser un potente antioxidante al actuar como secuestrador de radicales libres. Esto lo confirman estudios previos $(19,20,25)$, estableciendo con ello su reconocida actividad hipotensora, actividad que parece ser potenciada por el proceso de maceración del extracto de ajo. La demanda de tiempo implica mayores costos en la producción del extracto, tiempo que pretendió ser mejorado en este estudio.

La necesidad de reducir el tiempo de maceración de la extracción de ajo fue descrita por Sato et al. ${ }^{(17,18)}$, en donde expone que durante 40 días se pudo obtener metabolitos inherentes a su actividad antioxidante, mediante su técnica de fermentación espontanea, metabolitos derivados de la THBCs, como la metil- 
1,2,3,4-tetrahidro- $\beta$-carbolina-3-ácido-carboxílico (MTCC) sintetizada a partir del acetaldehído. Pese a que con este método no se ha determinado el efecto hipotensor in vivo, deja en claro la participación como secuestrador de radicales libres.

Indiscutiblemente, la propiedades ejercidas por el ajo sobre la presión arterial son atribuidas a la presencia de órganos sulfurados; en tal sentido, Asdaq y Inamda $2010^{(26)}$, demostraron una mejor eficacia terapéutica sobre la HTA al combinar ajo fresco con captopril que al serlo únicamente con SAC más captopril. En correlación con el presente estudio, se puede manifestar que cuando aumenta el tiempo de maceración del ajo se incrementa sus propiedades antioxidantes tanto como hipotensoras.

Aunque existen diversos métodos de procesamiento del ajo (fresco, calentado y añejado), parece que la diferencia entre estos métodos se basa en la cantidad de compuestos fenólicos, así como su bioestabilidad (27) y repercusión clínica ${ }^{(28)}$. Por ello, se recomienda un tratado adecuado combinando dichas técnicas de preparación ${ }^{(29)}$, así como evitar la biodegeneración por altas temperaturas, por lo tanto, un debido proceso de calentamiento y macerado podría incurrir en mejorar la cantidad de compuestos bioactivos y las propiedades antioxidantes e hipotensoras del ajo.

El estudio presenta algunas limitaciones que deben considerarse al momento de su replicación. Entre las principales se puede mencionar las siguientes: la falta de análisis químico mediante un marcador como principal indicador para la presencia de actividad hipotensora en el extracto de ajo añejado a mediano tiempo; el número de animales cumple un papel importante para extrapolar debidamente los resultados obtenidos a partir de la muestra $\mathrm{y}$, por último, el corto periodo de evaluación que en el presente estudio fue de 10 días; se presume que con un tiempo mayor o igual a 15 días el efecto hipotensor del ajo podría haber superado al del captopril.
Se recomienda explorar nuevas alternativas de técnicas de procesamiento del ajo, combinando los métodos antes descritos. Es lo que se pretendió en este estudio, empero, se deberá tener en cuenta superar las limitaciones que nos hemos enfrentado. A fin de lograr producir en una adecuada temperatura y tiempo de maceración, estructuras fenólicas en la cantidad y estabilidad necesaria, conllevando a reforzar los efectos terapéuticos del ajo. Pese a ser el presente estudio una inicial exploración in vivo, se deben continuar los esfuerzos ahora sobre los compuestos fenólicos (30), como base de futuros estudios.

Se concluye que el proceso de extracción del ajo en un plazo de 18 semanas de maceración en una dosis de $1000 \mathrm{mg} / \mathrm{kg}$ logra presentar una significativa reducción de la presión arterial en ratas con HTA por L-NAME, en un periodo de 10 días de tratamiento, siendo la única dosis tratada que no se evidencia diferencia significativa con el grupo captopril. En tal sentido, la dosis de 1000 $\mathrm{mg} / \mathrm{kg}$ de extracción de ajo a mediano plazo logra igualar los efectos hipotensores obtenidos en el grupo captopril.

Agradecimientos: a Rosa Velásquez Maguiña, por su importante colaboración durante la ejecución del presente estudio. Además, a cada una de las personas que formaron parte del equipo técnico haciendo realidad este estudio.

Contribuciones de autoría: DChM participó en la concepción, diseño del trabajo, interpretación de los datos y redacción de la primera versión del manuscrito. JR participó en la recolección y obtención de resultados y asesoría técnica, MG asesoría administrativa, y GFG participó en la redacción final y en la revisión crítica del artículo. Todos los autores aprobaron finalmente la última versión del artículo.

Fuente de financiamiento: el estudio fue completamente financiado por el Fondo Concursable de Investigación Científica del Vicerrectorado de Investigación, de la Universidad Peruana Cayetano Heredia. Identificado mediante código SIDISI: 58809.

Conflicto de interés: los autores declaran no tener conflictos de interés en la publicación de este artículo.

\section{REFERENCIAS BIBLIOGRÁFICAS}

1. Organización Mundial de la Salud. Prevención de las enfermedades crónicas [Internet]. Ginebra: OMS; 2013 [citado 7 de octubre de 2013]. Disponible en: http://www.who.int/ chp/chronic_disease_report/part1/es/ index.html

2. Boutayeb A. The double burden of communicable and non-communicable diseases in developing countries. Trans R Soc Trop Med Hyg. 2006 Mar;100(3):191-9.

3. Modesti PA, Agostoni P, Agyemang C, Basu S, Benetos A, Cappuccio FP, et al. Cardiovascular risk assessment in low-resource settings: a consensus document of the European Society of Hypertension Working Group on
Hypertension and Cardiovascular Risk in Low Resource Settings. J Hypertens. 2014 May;32(5):951-60. doi: 10.1097/ HJH.0000000000000125.

4. Segura Vega L, Agustí C. R, Ruiz Mori E. La hipertensión arterial en el Perú según el estudio TORNASOL II. Rev Peru Cardiol. 2011 EneAbr;37(1):19-27. 
5. Agusti R. Epidemiología de la Hipertensión Arterial en el Perú. Acta Med Per. 2006;23(2):69-75.

6. Davies AR, Miranda JJ, Gilman RH, Smeeth L. Hypertension among adults in a deprived urban area of Peru undiagnosed and uncontrolled? BMC Res Notes. 2008 Feb 26;1:2. doi: 10.1186/1756-0500-1-2.

7. Ayaz E, Alpsoy HC. [Garlic (Allium sativum) and traditional medicine]. Turkiye Parazitol Derg. 2007;31(2):145-9. [Article in Turkiish].

8. Harauma A, Moriguchi T. Aged garlic extract improves blood pressure in spontaneously hypertensive rats more safely than raw garlic. J Nutr. 2006 Mar;136(3 Suppl):769S-773S.

9. Yeh YY, Yeh SM. Homocysteinelowering action is another potential cardiovascular protective factor of aged garlic extract. J Nutr. 2006 Mar;136(3 Suppl):745S-749S

10. Cruz C, Correa-Rotter R, SánchezGonzález DJ, Hernández-Pando R, Maldonado PD, MartínezMartínez CM, et al. Renoprotective and antihypertensive effects of S-allylcysteine in 5/6 nephrectomized rats. Am J Physiol Renal Physiol. 2007 Nov;293(5):F1691-8.

11. Ried K, Frank OR, Stocks NP. Aged garlic extract reduces blood pressure in hypertensives: a dose-response trial. Eur J Clin Nutr. 2013 Jan;67(1):64-70. doi: 10.1038/ejcn.2012.178.

12. Ademiluyi AO, Oboh G, Owoloye TR, Agbebi OJ, Omonkhua AA. Modulatory effects of dietary inclusion of garlic (Allium sativum) on gentamycin-induced hepatotoxicity and oxidative stress in rats. Asian Pac J Trop Biomed. 2013 Jun;3(6):470-5. doi: 10.1016/S2221-1691(13)60098-2.

13. Nantz MP, Rowe CA, Muller CE, Creasy RA, Stanilka JM, Percival SS. Supplementation with aged garlic extract improves both NK and $\gamma \delta$-T cell function and reduces the severity of cold and flu symptoms: a randomized, double-blind, placebo-controlled nutrition intervention. Clin Nutr. 2012 Jun;31(3):337-44. doi: 10.1016/j. clnu.2011.11.019.
14. Weiss N, Papatheodorou L, Morihara N, Hilge R, Ide N. Aged garlic extract restores nitric oxide bioavailability in cultured human endothelial cells even under conditions of homocysteine elevation. J Ethnopharmacol. 2013 Jan 9;145(1):162-7. doi: 10.1016/j. jep.2012.10.045.

15. Morihara N, Ide N, Weiss N. Aged garlic extract inhibits homocysteineinduced scavenger receptor CD36 expression and oxidized lowdensity lipoprotein cholesterol uptake in human macrophages in vitro. J Ethnopharmacol. 2011 Apr 12;134(3):711-6. doi: 10.1016/j. jep.2011.01.021.

16. Aguilera P, Chánez-Cárdenas ME, Ortiz-Plata A, León-Aparicio D, Barrera $\mathrm{D}$, Espinoza-Rojo $\mathrm{M}$, et al. Aged garlic extract delays the appearance of infarct area in a cerebral ischemia model, an effect likely conditioned by the cellular antioxidant systems. Phytomedicine. 2010 Mar;17(3-4):241-7. doi: 10.1016/j.phymed.2009.06.004.

17. Sato E, Kohno M, Niwano Y. Increased level of tetrahydro-beta-carboline derivatives in short-term fermented garlic. Plant Foods Hum Nutr. 2006 Dec;61(4):175-8.

18. Sato E, Kohno M, Hamano H, Niwano Y. Increased anti-oxidative potency of garlic by spontaneous short-term fermentation. Plant Foods Hum Nutr. 2006 Dec;61(4):157-60.

19. Ichikawa M, Yoshida J, Ide N, Sasaoka T, Yamaguchi H, Ono K. Tetrahydro-betacarboline derivatives in aged garlic extract show antioxidant properties. J Nutr. 2006 Mar;136(3 Suppl):726S-731S.

20. Al-Qattan KK, Thomson M, AlMutawa’a S, Al-Hajeri D, Drobiova H, Ali M. Nitric oxide mediates the bloodpressure lowering effect of garlic in the rat two-kidney, one-clip model of hypertension. J Nutr. 2006 Mar;136(3 Suppl):774S-776S.

21. Pereira LM, Bezerra DG, Mandarimde-Lacerda CA. Aortic wall remodeling in rats with nitric oxide deficiency treated by enalapril or verapamil. Pathol Res Pract. 2004;200(3):211-7.

22. Rojas J, Ronceros S, Palomino R, Tomás G, Chenguayen J. Efecto antihipertensivo y dosis letal 50 del jugo del fruto y del extracto etanólico de las hojas de Passiflora edulis (maracuyá), en ratas. An Fac Med. 2006;67(3):206-13.

23. National Research Council. Guide for the Care and Use of Laboratory Animals. Washington, DC: The National Academies Press, 1996.

24. Whitley E, Ball J. Statistics review 3: hypothesis testing and $\mathrm{P}$ values. Crit Care. 2002 Jun;6(3):222-5.

25. Morihara N, Hayama M, Fujii H. Aged garlic extract scavenges superoxide radicals. Plant Foods Hum Nutr. 2011 Mar;66(1):17-21. doi: 10.1007/ s11130-011-0216-6.

26. Asdaq SM, Inamdar MN. Potential of garlic and its active constituent, S-allyl cysteine, as antihypertensive and cardioprotective in presence of captopril. Phytomedicine. 2010 Nov;17(13):1016-26. doi: 10.1016/j. phymed.2010.07.012.

27. Lawson LD, Gardner CD. Composition, stability, and bioavailability of garlic products used in a clinical trial. J Agric Food Chem. 2005 Aug;53(16):6254-61.

28. Milner JA. Preclinical perspectives on garlic and cancer. J Nutr. 2006 Mar;136(3 Suppl):827S-831S.

29. Capasso A. Antioxidant action and therapeutic efficacy of Allium sativum L. Molecules. 2013 Jan 4;18(1):690-700. doi: 10.3390/ molecules18010690.

30. Bozin B, Mimica-Dukic N, Samojlik I, Goran A, Igic R. Phenolics as antioxidants in garlic (Allium sativum L., Alliaceae). Food Chem. 2008 Dec;111(4):925-9.

Correspondencia: David Chaupis Meza Dirección: Universidad Peruna Cayetano Heredia, Av. Honorio Delgado 430. Lima 31, Perú.

Telefono: 948496320

Correo electrónico:david.chaupis.m@upch.pe 